\section{Advanced paternal age increases risk of bipolar disorder in offspring}

\section{QUESTION}

Question: Does advanced paternal age increase the risk of bipolar disorder in offspring?

People: 7739202 individuals were identified through linkage of the Hospital Discharge Register and the Multigeneration Register. From this study base of over 7 million individuals, 13428 cases (42\% male) with two known biological parents and at least two separate hospital admissions for bipolar disorder (using ICD codes) were identified. Exclusions: people diagnosed with bipolar disorder but with only one hospitalisation (to avoid inclusion of people who were misdiagnosed); people diagnosed with unipolar depression. Controls (67 140 people; five randomly selected for each case) were people without a diagnosis of bipolar disorder and matched to cases for sex and age. Controls had to be alive at the date of the case's first hospitalisation to allow for an equal period of risk.

Setting: General population, Sweden; registry data was obtained for the period 1932 and 1991.

Risk factors: The primary risk factor of interest was paternal age. Conditional logistic regression analysis was used to estimate risk of bipolar disorder controlling for possible confounding factors of maternal age, parity, parental family history of psychotic disorders and socioeconomic status. People with early onset bipolar disorder (diagnosed when aged $<20$ years) were analysed separately to assess if there was any difference in risk. The effects of maternal age on risk of bipolar disorder in offspring were also examined.

Outcomes: Bipolar disorder (defined as diagnosis of bipolar disorder at hospital discharge on two or more occasions).

\section{METHODS}

Design: Nested case control study.

Follow-up period: Up to 59 years (assessed retrospectively).

\section{MAIN RESULTS}

The risk of bipolar disorder in the offspring increased with advancing paternal age (see online table). Compared with offspring of men aged 20-24 years, the risk of bipolar disorder was highest in offspring of men aged 55 years and older (unadjusted odds ratio (OR) 1.38, 95\% CI 1.04 to 1.84). This increase remained significant after adjustment for a family history of psychotic disorders, maternal age, socioeconomic status and parity (OR 1.37, 95\% CI 1.02 to 1.84). For cases with early onset bipolar disorder (715 cases and 3575 controls included in the analysis), the risk of bipolar disorder was highest in offspring of men aged 50 years and older compared with offspring of men aged 20-24 years (OR 2.63, $95 \%$ CI 1.19 to 5.81; adjusted for maternal age). Increasing maternal age was also associated with an increased risk of bipolar disorder. However, after controlling for paternal age, family history of psychotic disorders, parity and socioeconomic status, the increase only remained significantly higher in offspring of women aged 30-34 years (OR 1.08, 95\% CI 1.01 to 1.16 ) and 35-39 years (OR 1.16, $95 \%$ CI 1.06 to 1.26 ) compared with offspring of women aged 20-24 years. After adjusting for paternal age, the associations between maternal age and risk for early onset bipolar disorder were not statistically significant (OR 1.24, 95\% CI 0.71 to 2.19 ).

\section{CONCLUSIONS}

Advanced paternal age is a risk factor for bipolar disorder in offspring.

\section{ABSTRACTED FROM}

Frans EM, Sandin S, Reichenberg A, et al. Advancing paternal age and bipolar disorder. Arch Gen Psychiatry 2008;65:1034-40.

Correspondence to: Emma M Frans, MMedSc, Department of Medical Epidemiology and Biostatistics, Karolinksa Institute, P0 Box 281, SE-171 77 Stockholm, Sweden; Emma.Frans@ki.se

Source of funding: None reported.

- A table is published online only at http://ebmh.bmj.com/content/vol12/issue2
A dvanced paternal age has historically been examined in association with psychiatric disease in the offspring. However, most studies have suffered from methodological shortcomings and the "new wave" started in 2001 when Malaspina and colleagues, based on a large cohort, described an almost three times increased risk for the offspring to develop schizophrenia if the father was aged 50 years or older at the time of birth of the child. ${ }^{1}$ Since then at least six more studies have been published on the subject, all showing a doseresponse relationship. Recently, an association with another neurodevelopmental disorder, autism spectrum disorder (ASD), was described. ${ }^{2}{ }^{3}$ Now Frans and colleagues present evidence of an association between paternal age and risk of bipolar disorder (BPD). There was a stepwise increased risk and offspring of fathers aged 55 years and older had an increased risk of 1.38. This is a somewhat lower risk estimate than those described in previous studies of schizophrenia. However, in subanalysis of BPD cases with "early onset", defined as hospitalised at an early age ( $<20$ years), the effect was stronger (OR 2.63).

In summary, there is now evidence of an association between advancing paternal age and risk of BPD, ASD and schizophrenia. This is intriguing and the question is, what do these psychiatric disorders have in common? One thing is a substantial genetic component. Two hypotheses about the underlying mechanisms of the paternal age effect are currently discussed: de novo mutations is one such hypothesis. In the production of sperms, the germ cells replicate regularly resulting in an increasing number of divisions by advancing age and thus the risk for DNA copy errors increases. Another hypothesis is that aberrant epigenetic regulation explains the association. Both of these mechanisms may lead to heritable changes and can explain how the illnesses are maintained in the population given the reduced fertility of those with some of the disorders.

\section{Christina Dalman, MD, PhD}

Department of Public Health Sciences, Karolinska Institutet., Stockholm, Sweden

\section{Competing interests: None.}

1. Malaspina D, Harlap S, Fennig S, et al. Advancing paternal age and the risk of schizophrenia. Arch Gen Psychiatry 2001;58:361-7.

2. Reichenberg A, Gross R, Weiser M, et al. Advancing paternal age and autism. Arch Gen Psychiatry 2006;63: 1026-32.

3. Croen LA, Najjar DV, Fireman B, et al. Maternal and paternal age and risk of autism spectrum disorders. Arch Pediatr Adolesc Med 2007;161:334-40. 